\title{
Aktive Immuntherapie auf dem Prüfstand
}

Fragestellung: Die aktive Immuntherapie gegen das Amyloid $\beta(A \beta)$ ist eine mögliche therapeutische Strategie gegen die Alzheimer-Demenz (AD).

Hintergrund: $\mathrm{A} \beta$ ist entscheidend an der Iniitierung der AD beteiligt. Eine erste Studie mit aktiver Immunisierung (AN1792) scheiterte an einer T-Zell vermittelten Meningoenzephalitis, die in $6 \%$ der Fälle auftrat. Das Prinzip der Immunisation konnte bestätigt werden, da die Patienten $A \beta$-Antikörper aufbauten und $\mathrm{A} \beta$-Belastung, funktioneller Abbau und die Angehörigenbelastung abnahmen. Der kognitive Abbau konnte jedoch kaum verlangsamt werden. Während sich AN1792 gegen das gesamte $A \beta$ Peptid (1 - 42) richtete, ist CAD106 ein Impfstoff gegen das A $\beta$ Fragment 1-6 und soll eine B-Zellantwort hervorrufen.

Patienten und Methodik: Die Phase-I-Studie randomisierte 58 Patienten (50 bis 80 Jahre mit leichter bis moderater AD) im Verhältnis 4:1 (Verum : Placebo). Die erste Kohorte $(\mathrm{n}=31)$ erhielt $50 \mu \mathrm{g}$ CAD106, die zweite Kohorte $(\mathrm{n}=27) 150 \mu \mathrm{g} 3$ x s.c. Studienziele waren Sicherheit, Verträglichkeit und die Anti-

Winblad B, Andreasen N, Minthon L et al. Safety, tolerability, and antibody response of active $A \beta$ immunotherapy with CAD106 in patients with Alzheimer's disease: randomised, double-blind, placebo-controlled, first-in-human study. Lancet 2012; 11: 597-604 körperantwort gegen $A \beta$.

Ergebnisse: Nahezu alle Patienten (56 von 58 ) berichteten Nebenwirkungen, meist Nasopharyngitis, Fieber und ein Erythem an der Injektionsstelle. Eine Meningoenzephalitis trat nicht auf. In

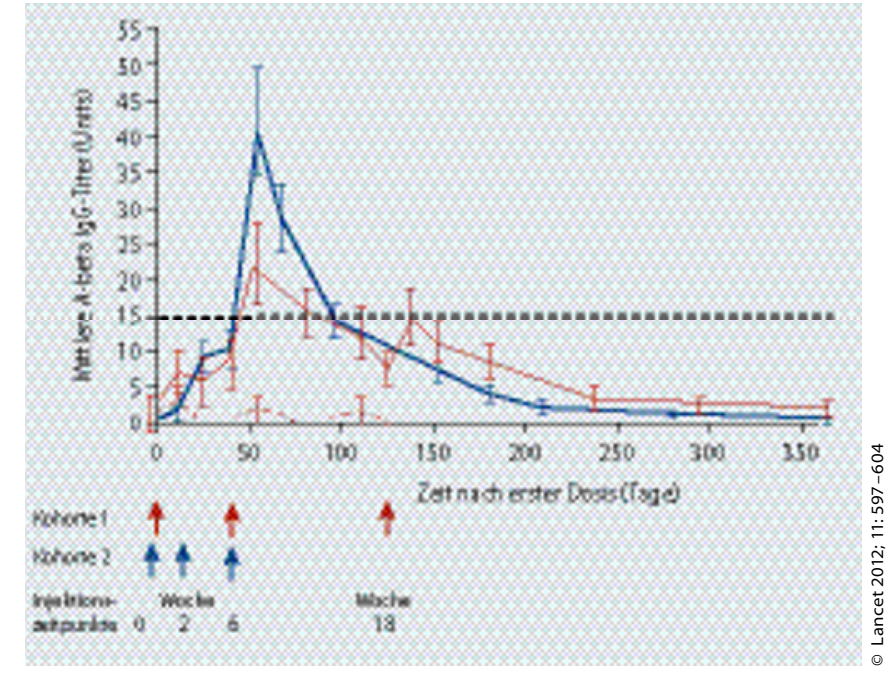

1 Aß-spezifische Antikörperantwort (IgG-Titer).

Kohorte 2 entwickelten 18 von 22 Patienten (82\%) eine Antikörperantwort, in Kohorte 1 etwas weniger. Das Maximum der Antikörperantwort trat nach acht Wochen auf. Die an Antikörper gebundene $\mathrm{A} \beta$-Konzentration stieg an.

Schlussfolgerungen: CAD106 hat ein günstiges Sicherheitsprofil und eine vielversprechende Antikörperantwort, die möglicherweise durch weitere Dosisfindungsstudien verbessert werden kann.

\section{-Kommentar von Prof. Markus Weih}

\section{Es gibt noch einiges zu tun}

Diese klassische kleine Phase I, first-in-human-Studie belegt methodisch hochwertig (Jadad-Score 5), dass CAD106 ein günstiges Sicherheits- und Verträglichkeitsprofil aufweist. Insbesondere traten keine autoimmunologischen Nebenwirkungen auf, was bei einer Impfung gegen ein ubiquitäres ZNSProtein wie $A \beta$ alleine bemerkenswert ist. Die Mehrzahl der Patienten entwickelte $A \beta$-Antikörper, die auch wirksam $A \beta$ im Plasma banden.

Im Liquor zeigten sich keine Veränderungen der Biomarker nach Impfung, was bei der relativ kurzen Zeit auch noch nicht zu erwarten war. Natürlich kann über den eigentlichen therapeutischen Effekt in Phase I noch keine Aussage getroffen werden. Da sich die $A \beta$-Pathologie über Jahrzehnte entwickelt und im Verlauf der Krankheit die Neurodegeneration und die Tau-Pathologie zunehmen, wird es sicher noch Jahre dauern, bis endgültige Aussagen über die Wirksamkeit einer aktiven Impfung gegen die AD getroffen werden können. Nach dem Scheitern der Sekretasestudien gibt es weiter leider keine kurz- fristige neue Therapieoption. Die meisten Cholinesterasehemmer und Memantine sind als Generika erhältlich, werden in Leitlinien empfohlen, aber in Deutschland zu selten eingesetzt. Es gibt also noch einiges zu tun. Die Impfung wird für die aktuelle Generation der AD-Patienten keine Option darstellen.

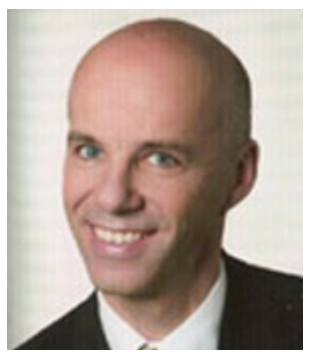

Prof. Dr. med. Markus Weih, Nürnberg

Facharzt für Neurologie, Psychiatrie und

Psychotherapie, Nervenärztliche

Gemeinschaftspraxis, Nürnberg

E-Mail: weih@nervenaerzte-

allersbergerstrasse.de 